\title{
ITERATED SEQUENCES AND THE GEOMETRY OF ZEROS
}

\author{
PETTER BRÄNDÉN \\ Dedicated to the memory of Julius Borcea
}

\begin{abstract}
We study the effect on the zeros of generating functions of sequences under certain non-linear transformations. Characterizations of PólyaSchur type are given of the transformations that preserve the property of having only real and non-positive zeros. In particular, if a polynomial $a_{0}+a_{1} z+$ $\cdots+a_{n} z^{n}$ has only real and non-positive zeros, then so does the polynomial $a_{0}^{2}+\left(a_{1}^{2}-a_{0} a_{2}\right) z+\cdots+\left(a_{n-1}^{2}-a_{n-2} a_{n}\right) z^{n-1}+a_{n}^{2} z^{n}$. This confirms a conjecture of Fisk, McNamara-Sagan and Stanley, respectively. A consequence is that if a polynomial has only real and non-positive zeros, then its Taylor coefficients form an infinitely log-concave sequence. We extend the results to transcendental entire functions in the Laguerre-Pólya class, and discuss the consequences to problems on iterated Turán inequalities, studied by Craven and Csordas. Finally, we propose a new approach to a conjecture of Boros and Moll.
\end{abstract}

\section{Contents}

1. Introduction

2. Symmetric function identities

3. Grace-Walsh-Szegő type theorems and a proof of Conjecture 1.1

4. Algebraic Pólya-Schur characterizations of transformations

5. Transcendental Pólya-Schur characterizations of transformations

6. Applications and examples

7. Refined results on the location of zeros

8. Iterated Turán inequalities and the Boros-Moll conjecture 13

References

\section{INTRODUCTION}

Let $\Phi$ be a transformation of sequences of real numbers, and let $\left\{a_{k}\right\}$ be a real sequence. We are interested in when the iterates $\Phi^{i}\left(\left\{a_{k}\right\}\right)$, for $i \in \mathbb{N}$, are nonnegative. Such questions appear in the theory of entire functions [4, 5], and recently in the theory of special functions [2, 7, 8] and combinatorics [10, 17]. It has been made evident that the zero set of the generating function of $\left\{a_{k}\right\}$ plays a prominent

2000 Mathematics Subject Classification. 26C10, 30C15, 05A20.

Key words and phrases. infinite log-concavity, distribution of zeros, real zeros, entire functions, Laguerre-Pólya class, Turán inequalities.

Supported by the Göran Gustafsson Foundation.

To appear in J. Reine Angew. Math. (Crelle's journal). 
role in such questions. One purpose of this paper is to make this correspondence explicit.

Let $\left\{a_{k}\right\}=\left\{a_{k}\right\}_{k=0}^{n}$, where $n \in \mathbb{N} \cup\{\infty\}$, be a sequence of real numbers. The sequence is log-concave if $a_{k}^{2}-a_{k-1} a_{k+1} \geq 0$, for all $1 \leq k \leq n-1$. Define an operator on sequences by $\mathcal{L}\left(\left\{a_{k}\right\}\right)=\left\{b_{k}\right\}_{k=0}^{n}$, where $b_{k}=a_{k}^{2}-a_{k-1} a_{k+1}$ for all $0 \leq k \leq n$, and $a_{-1}=a_{n+1}=0$. Hence, $\left\{a_{k}\right\}$ is log-concave if and only if $\mathcal{L}\left(\left\{a_{k}\right\}\right)$ is non-negative. The sequence is $i$-fold log-concave if the $i$ th iterate, $\mathcal{L}^{i}\left(\left\{a_{k}\right\}\right)$, is non-negative, and infinitely log-concave if it is $i$-fold log-concave for all $i \in \mathbb{N}$. Boros and Moll [2] conjectured that the sequence of binomial numbers, $\left\{\left(\begin{array}{l}n \\ k\end{array}\right)\right\}_{k=0}^{n}$, is infinitely $\log$-concave for each $n \in \mathbb{N}$. If the polynomial $\sum_{k=0}^{n} a_{k} z^{k}$ has only real and non-positive zeros, then it follows that the sequence $\left\{a_{k}\right\}$ is log-concave. Motivated by this fact and Boros and Moll's conjecture on binomial numbers, Stanley [17, McNamara-Sagan [10] and Fisk [7, independently made the following conjecture.

Conjecture 1.1. Suppose that the polynomial $\sum_{k=0}^{n} a_{k} z^{k}$ has only real and negative zeros. Then so does the polynomial

$$
\sum_{k=0}^{n}\left(a_{k}^{2}-a_{k-1} a_{k+1}\right) z^{k}, \quad \text { where } a_{-1}=a_{n+1}=0 .
$$

In particular, the sequence $\left\{a_{k}\right\}_{k=0}^{n}$ is infinitely log-concave.

It should be mentioned that similar questions were raised already in [4, 5], see Section 8 In Section 3 we prove Conjecture 1.1. However, we take a general approach and study a large class of transformations of sequences. Let $\alpha=\left\{\alpha_{k}\right\}_{k=0}^{\infty}$ be a fixed sequence of complex numbers and define two sequences $\mathcal{L}_{\alpha}^{E}\left(\left\{a_{k}\right\}_{k=0}^{n}\right)=$ $\left\{b_{k}(\alpha)\right\}_{k=0}^{n}$ and $\mathcal{L}_{\alpha}^{O}\left(\left\{a_{k}\right\}_{k=0}^{n}\right)=\left\{c_{k}(\alpha)\right\}_{k=0}^{n}$, where

$$
b_{k}(\alpha)=\sum_{j=0}^{\infty} \alpha_{j} a_{k-j} a_{k+j} \quad \text { and } \quad c_{k}(\alpha)=\sum_{j=0}^{\infty} \alpha_{j} a_{k-j} a_{k+1+j},
$$

and $a_{j}=0$ if $j \notin\{0, \ldots, n\}$. In Theorems 5.7 and 5.8 we characterize the sequences $\alpha$, for which $\mathcal{L}^{E}$ (or $\mathcal{L}^{O}$ ) preserves the property of having generating polynomial with only real and non-positive zeros. The characterization is of Pólya-Schur type; that is, $\mathcal{L}_{\alpha}^{E}$ (or $\mathcal{L}_{\alpha}^{O}$ ) has the desired properties if and only if the generating function of $\mathcal{L}_{\alpha}^{E}(\{1 / k !\})$ (or $\left.\mathcal{L}_{\alpha}^{O}(\{1 / k !\})\right)$ is an entire function that can be approximated, uniformly on compact subsets of $\mathbb{C}$, by polynomials with only negative zeros. Similar characterizations of classes of transformations were given in [12] and [1]. The fundamental difficulty in our setting is that in 1, 12, the transformations in question are linear, whereas the transformations that we consider are not. This potential problem is overcome by a symmetric function identity (Theorem 2.1) that linearizes the problem.

In Section 8 we propose a new approach to the original conjecture (see Conjecture 8.4) of Boros and Moll [2]. We state a conjecture that would imply 3-fold logconcavity of the sequences in question.

\section{Symmetric Function identities}

Let $\left\{e_{k}(\mathbf{z})\right\}_{k=0}^{n}$ denote the elementary symmetric functions in the variables $\mathbf{z}=$ $\left(z_{1}, \ldots, z_{n}\right)$, and set $e_{k}(\mathbf{z})=0$ for $k \notin\{0, \ldots, n\}$. If $\mu=\left\{\mu_{k}\right\}_{k=0}^{\infty}$ is a sequence of 
complex numbers, we define a symmetric function by

$$
W_{\mu, n}(\mathbf{z})=\sum_{i \leq j} \mu_{j-i} e_{i}(\mathbf{z}) e_{j}(\mathbf{z}) .
$$

Theorem 2.1. Let $\mu=\left\{\mu_{k}\right\}_{k=0}^{\infty}$ be a sequence of complex numbers, and let

$$
\gamma_{k}=\sum_{j=0}^{\lfloor k / 2\rfloor}\left(\begin{array}{l}
k \\
j
\end{array}\right) \mu_{k-2 j}, \quad \text { for } k \in \mathbb{N} .
$$

Then

$$
W_{\mu, n}(\mathbf{z})=e_{n}(\mathbf{z}) \sum_{k=0}^{n} \gamma_{k} e_{n-k}\left(\mathbf{z}+\frac{1}{\mathbf{z}}\right),
$$

where $1 / \mathbf{z}=\left(1 / z_{1}, \ldots, 1 / z_{n}\right)$.

Proof. By linearity it is enough to prove the theorem for the case when there is a number $m \in \mathbb{N}$ such that $\mu_{m}=1$, and $\mu_{k}=0$ for each $k \neq m$.

Following [3, 11], for $k, r, n \in \mathbb{N}$, define a symmetric function $\sigma_{k}^{r}(\mathbf{z})$ by

$$
\sigma_{k}^{r}(\mathbf{z})=\sum_{\alpha=\left(\alpha_{1}, \ldots, \alpha_{n}\right)} z_{1}^{\alpha_{1}} \cdots z_{n}^{\alpha_{n}},
$$

where the summation is over all $\alpha \in\{0,1,2\}^{n}$ such that $\alpha_{1}+\cdots+\alpha_{n}=k$, and $\left|\left\{i: \alpha_{i}=2\right\}\right|=r$. By a simple counting argument, see [3, 11,

$$
e_{i}(\mathbf{z}) e_{j}(\mathbf{z})=\sum_{r}\left(\begin{array}{c}
i-r+j-r \\
i-r
\end{array}\right) \sigma_{i+j}^{r}(\mathbf{z}),
$$

and thus

$$
e_{i}(\mathbf{z}) e_{i+m}(\mathbf{z})=\sum_{j}\left(\begin{array}{c}
2 j+m \\
j
\end{array}\right) \sigma_{m+2 i}^{i-j}(\mathbf{z}) .
$$

From the definition of $\sigma_{m+2 i}^{i-j}(\mathbf{z})$ we see that

$$
\sigma_{m+2 i}^{i-j}(\mathbf{z})=\sum_{|S|=m+2 j} \mathbf{z}^{S} e_{i-j}\left(z_{t}^{2}: t \notin S\right),
$$

where $\mathbf{z}^{S}=\prod_{s \in S} z_{s}$. Summing over all $i$ in the equation above yields

$$
\sum_{|S|=m+2 j} \mathbf{z}^{S} \prod_{t \notin S}\left(1+z_{t}^{2}\right)=e_{n}(\mathbf{z}) e_{n-m-2 j}\left(z_{1}+\frac{1}{z_{1}}, \ldots, z_{n}+\frac{1}{z_{n}}\right) .
$$

Equation (2), for our choice of $\mu$, now follows from (44) when summing over all $j$.

We pause here to sketch an alternative combinatorial proof of the important case of (2) when $\mu=\{1,0,-1,0,0, \ldots\}$. For undefined symmetric function terminology we refer to [16, Chapter 7]. For our particular choice of $\mu$, we want to prove the identity

$$
\sum_{k=0}^{n}\left(e_{k}(\mathbf{z})^{2}-e_{k-1}(\mathbf{z}) e_{k+1}(\mathbf{z})\right)=e_{n}(\mathbf{z}) \sum_{k=0}^{\lfloor n / 2\rfloor} C_{k} e_{n-2 k}\left(\mathbf{z}+\frac{1}{\mathbf{z}}\right)
$$


where $C_{k}=\left(\begin{array}{c}2 k \\ k\end{array}\right) /(k+1)$ is a Catalan number, see [16, Exercise 6.19]. We may rewrite (5) as

$$
\sum_{k=0}^{n}\left(e_{k}(\mathbf{z})^{2}-e_{k-1}(\mathbf{z}) e_{k+1}(\mathbf{z})\right)=\sum_{k=0}^{\lfloor n / 2\rfloor} C_{k} \sum_{|S|=2 k} \mathbf{z}^{S} \prod_{j \notin S}\left(1+z_{j}^{2}\right) .
$$

The polynomial $e_{k}(\mathbf{z})^{2}-e_{k-1}(\mathbf{z}) e_{k+1}(\mathbf{z})$ is the Schur-function $s_{2^{k}}(\mathbf{z})$, where $2^{k}=$ $(2,2, \ldots, 2)$. By the combinatorial definition of the Schur-function, the left hand side of (6) is the generating polynomial of all semi-standard Young tableaux with entries in $\{1, \ldots, n\}$, that are of shape $2^{k}$ for some $k \in \mathbb{N}$. Call this set $\mathcal{A}_{n}$. Given $T \in \mathcal{A}_{n}$, let $S$ be the set of entries which occur only ones in $T$. By deleting the remaining entries we obtain a standard Young tableau of shape $2^{k}$, where $2 k=|S|$. There are exactly $C_{k}$ standard Young tableaux of shape $2^{k}$ with set of entries $S$, see e.g. [16, Exercise 6.19.ww]. The original semi-standard Young tableau is then determined by the set of duplicates. This explains the right hand side of (6).

\section{Grace-Walsh-Szegö type theorems and a proof of Conjecture 1.1}

The Grace-Walsh-Szegö Theorem is undoubtably one of the most useful theorems governing the location of zeros of polynomials, see [13. A circular region is a proper subset of the complex plane that is bounded by either a circle or a straight line, and is either open or closed. A polynomial is multi-affine provided that each variable occurs at most to the first power.

Theorem 3.1 (Grace-Walsh-Szegö). Let $f \in \mathbb{C}\left[z_{1}, \ldots, z_{n}\right]$ be a multi-affine and symmetric polynomial, and let $K$ be a circular region. Assume that either $K$ is convex or that the degree of $f$ is $n$. For any $\zeta_{1}, \ldots, \zeta_{n} \in K$ there is a $\zeta \in K$ such that $f\left(\zeta_{1}, \ldots, \zeta_{n}\right)=f(\zeta, \ldots, \zeta)$.

We are now in a position to prove Conjecture 1.1.

Proof of Conjecture 1.1. Let $P(z)=\sum_{k=0}^{n} a_{k} z^{k}=\prod_{k=0}^{n}\left(1+\rho_{k} z\right)$, where $\rho_{k}>0$ for all $1 \leq k \leq n$, and let

$$
Q(z)=\sum_{k=0}^{n}\left(a_{k}^{2}-a_{k-1} a_{k+1}\right) z^{k} .
$$

Suppose that there is a $\zeta \in \mathbb{C}$, with $\zeta \notin\{x \in \mathbb{R}: x \leq 0\}$, for which $Q(\zeta)=0$. We may write $\zeta$ as $\zeta=\xi^{2}$, where $\operatorname{Re}(\xi)>0$. By (5),

$$
0=Q(\zeta)=a_{n} \xi^{n} \sum_{k=0}^{\lfloor n / 2\rfloor} C_{k} e_{n-2 k}\left(\rho_{1} \xi+\frac{1}{\rho_{1} \xi}, \ldots, \rho_{n} \xi+\frac{1}{\rho_{n} \xi}\right),
$$

where $C_{k}=\left(\begin{array}{c}2 k \\ k\end{array}\right) /(k+1)$. Since $\operatorname{Re}\left(\rho_{j} \xi+1 /\left(\rho_{j} \xi\right)\right)>0$ for all $1 \leq j \leq n$, the Grace-Walsh-Szegö Theorem provides a $\eta \in \mathbb{C}$, with $\operatorname{Re}(\eta)>0$, such that

$$
0=\sum_{k=0}^{\lfloor n / 2\rfloor} C_{k} e_{n-2 k}(\eta, \ldots, \eta)=\sum_{k=0}^{\lfloor n / 2\rfloor} C_{k}\left(\begin{array}{c}
n \\
2 k
\end{array}\right) \eta^{n-2 k}=: \eta^{n} p_{n}\left(\frac{1}{\eta^{2}}\right) .
$$

Since $\operatorname{Re}(\eta)>0$, we have $1 / \eta^{2} \in \mathbb{C} \backslash\{x \in \mathbb{R}: x \leq 0\}$. Hence, the desired contradiction follows if we can prove that all the zeros of $p_{n}(z)$ are real and negative. 
This follows from the identity

$$
\begin{aligned}
\sum_{k=0}^{\lfloor n / 2\rfloor} C_{k}\left(\begin{array}{c}
n \\
2 k
\end{array}\right) z^{k}(1+z)^{n-2 k} & =\sum_{k=0}^{n} \frac{1}{n+1}\left(\begin{array}{c}
n+1 \\
k
\end{array}\right)\left(\begin{array}{c}
n+1 \\
k+1
\end{array}\right) z^{k} \\
& =\frac{1}{n+1}(1-z)^{n} P_{n}^{(1,1)}\left(\frac{1+z}{1-z}\right),
\end{aligned}
$$

where $\left\{P_{n}^{(1,1)}(z)\right\}_{n}$ are Jacobi polynomials, see [14, p. 254]. The zeros of the Jacobi polynomials $\left\{P_{n}^{(1,1)}(z)\right\}_{n}$ are located in the interval $(-1,1)$. Note that the first identity in the equation above follows immediately from (5).

Now that Conjecture 1.1 is established we shall see how the ideas in the proof can be extended considerably.

If $\mu$ is a sequence of complex numbers, define a (non-linear) operator, $T_{\mu}: \mathbb{C}[z] \rightarrow$ $\mathbb{C}[z]$, by

$$
T_{\mu}\left(\sum_{k=0}^{n} a_{k} z^{k}\right)=\sum_{i \leq j} \mu_{j-i} a_{i} a_{j} z^{i+j} .
$$

Define polynomials, $P_{\mu, n}(z)$, for $n \in \mathbb{N}$, by

$$
P_{\mu, n}(z)=\sum_{k=0}^{n} \gamma_{k}\left(\begin{array}{l}
n \\
k
\end{array}\right) z^{n-k}=\sum_{j, k}\left(\begin{array}{l}
n \\
k
\end{array}\right)\left(\begin{array}{l}
k \\
j
\end{array}\right) \mu_{k-2 j} z^{n-k} .
$$

A complex polynomial $F\left(z_{1}, \ldots, z_{n}\right)$ is weakly Hurwitz stable if $F\left(z_{1}, \ldots, z_{n}\right) \neq 0$ whenever $\operatorname{Re}\left(z_{j}\right)>0$ for all $1 \leq j \leq n$. The following theorem can be seen as a Grace-Walsh-Szegö theorem for certain non-multi-affine polynomials.

Theorem 3.2. Let $\mu$ be a sequence of complex numbers. The following are equivalent.

(i) $W_{\mu, n}(\mathbf{z})$ is weakly Hurwitz stable;

(ii) For all polynomials $P(z)$ of degree at most n, with only real and non-positive zeros, the polynomial $T_{\mu}(P(z))$ is either identically zero or weakly Hurwitz stable;

(iii) $T_{\mu}\left((1+z)^{n}\right)=W_{\mu, n}(z, \ldots, z)$ is weakly Hurwitz stable;

(iv) The polynomial $P_{\mu, n}(z)$ is weakly Hurwitz stable.

Proof. Suppose that $W_{\mu, n}(\mathbf{z})$ is weakly Hurwitz stable, and that $P(z)$ is a real polynomial of degree at most $n$ with only real and non-positive zeros. By Hurwitz' theorem on the continuity of zeros, see e.g. [13, Theorem 1.3.8], we may assume that $P(z)=\prod_{j=1}^{n}\left(1+\rho_{j} z\right)$, where $\rho_{j}>0$ for all $1 \leq j \leq n$. Suppose that $\operatorname{Re}(\zeta)>0$. Then $\operatorname{Re}\left(\rho_{j} \zeta\right)>0$, for all $1 \leq j \leq n$. Hence

$$
W_{\mu, n}\left(\rho_{1} \zeta, \ldots, \rho_{n} \zeta\right)=T_{\mu}(P(\zeta)) \neq 0
$$

which proves (i) $\Rightarrow$ (ii).

The implication (ii) $\Rightarrow$ (iii) is obvious. Clearly, by (2),

$$
W_{\mu, n}(z, \ldots, z)=z^{n} P_{\mu, n}\left(z+\frac{1}{z}\right) .
$$

Hence, the equivalence of (iii) and (iv) follows from the set identity

$$
\{z+1 / z: z \in \mathbb{C} \text { and } \operatorname{Re}(z)>0\}=\{z \in \mathbb{C}: \operatorname{Re}(z)>0\} .
$$


Now, suppose that $W_{\mu, n}\left(\zeta_{1}, \ldots, \zeta_{n}\right)=0$, where $\operatorname{Re}\left(\zeta_{j}\right)>0$ for all $1 \leq j \leq n$. Then, by (2),

$$
\sum_{k=0}^{n} \gamma_{k} e_{n-k}\left(\zeta_{1}+\frac{1}{\zeta_{1}}, \ldots, \zeta_{n}+\frac{1}{\zeta_{n}}\right)=0 .
$$

Since $\operatorname{Re}\left(\zeta_{j}+1 / \zeta_{j}\right)>0$, for all $1 \leq j \leq n$, the Grace-Walsh-Szegö theorem provides a number $\xi \in \mathbb{C}$, with $\operatorname{Re}(\xi)>0$, such that

$$
0=\sum_{k=0}^{n} \gamma_{k} e_{n-k}(\xi, \ldots, \xi)=P_{\mu, n}(\xi) .
$$

This verifies (iv) $\Rightarrow$ (i).

\section{Algebraic Pólya-Schur characterizations of transformations}

Let us turn to the cases when all the non-zero $\mu_{i}$ 's have the same parity. Let $\alpha=\left\{\alpha_{k}\right\}_{k=0}^{\infty}$ be a fixed sequence of complex numbers and define two sequences $\mathcal{L}_{\alpha}^{E}\left(\left\{a_{k}\right\}_{k=0}^{n}\right)=\left\{b_{k}(\alpha)\right\}_{k=0}^{n}$ and $\mathcal{L}_{\alpha}^{O}\left(\left\{a_{k}\right\}_{k=0}^{n}\right)=\left\{c_{k}(\alpha)\right\}_{k=0}^{n}$, where

$$
b_{k}(\alpha)=\sum_{j=0}^{\infty} \alpha_{j} a_{k-j} a_{k+j} \quad \text { and } \quad c_{k}(\alpha)=\sum_{j=0}^{\infty} \alpha_{j} a_{k-j} a_{k+1+j},
$$

and $a_{j}=0$ if $j \notin\{0,1, \ldots, n\}$. Define also two non-linear operators on polynomials, $U_{\alpha}, V_{\alpha}: \mathbb{C}[z] \rightarrow \mathbb{C}[z]$, by

$$
U_{\alpha}\left(\sum_{k=0}^{n} a_{k} z^{k}\right)=\sum_{k=0}^{n} b_{k}(\alpha) z^{k} \quad \text { and } \quad V_{\alpha}\left(\sum_{k=0}^{n} a_{k} z^{k}\right)=\sum_{k=0}^{n} c_{k}(\alpha) z^{k} .
$$

We want to characterize the real sequences $\alpha$ for which $U_{\alpha}$ (or $V_{\alpha}$ ) send polynomials with only real and non-positive zeros to polynomials of the same kind.

If $P(z)=\sum_{k=0}^{n} a_{k} z^{k}$, let

$$
P^{E}(z)=\sum_{k=0}^{\lfloor n / 2\rfloor} a_{2 k} z^{k} \quad \text { and } \quad P^{O}(z)=\sum_{k=0}^{\lfloor(n-1) / 2\rfloor} a_{2 k+1} z^{k} .
$$

The next theorem is a version of the classical Hermite-Biehler theorem, see e.g. [13, p. 197].

Theorem 4.1 (Hermite-Biehler). Let $P(z)=P^{E}\left(z^{2}\right)+z P^{O}\left(z^{2}\right) \in \mathbb{R}[z]$. Then $P(z)$ is weakly Hurwitz stable if and only if all non-zero coefficients of $P$ have the same sign, and

- $P^{E}(z) \equiv 0$, and $P^{O}(z)$ has only real and non-positive zeros, or

- $P^{O}(z) \equiv 0$, and $P^{E}(z)$ has only real and non-positive zeros, or

- $P^{E}(z) P^{O}(z) \not \equiv 0$, and $P^{E}(z)$ and $P^{O}(z)$ have real and non-positive zeros which are interlacing in the following sense. If $z_{m}^{\prime} \leq \cdots \leq z_{1}^{\prime}$ and $z_{\ell} \leq$ $\cdots \leq z_{1}$ are the zeros of $P^{E}(z)$ and $P^{O}(z)$, respectively, then

$$
\cdots \leq z_{3} \leq z_{2}^{\prime} \leq z_{2} \leq z_{1}^{\prime} \leq z_{1} .
$$

Given a sequence $\mu$, we define two auxiliary operators, $T_{\mu}^{E}, T_{\mu}^{O}: \mathbb{C}[z] \rightarrow \mathbb{C}[z]$, by

$$
T_{\mu}^{E}(P(z))=T_{\mu}(P(z))^{E} \quad \text { and } \quad T_{\mu}^{O}(P(z))=T_{\mu}(P(z))^{O} .
$$

Let $\mathcal{P}_{n}^{+}$denote the set of all polynomials of degree at most $n$ with only real and non-positive zeros, and let $\mathcal{P}^{+}=\bigcup_{n=0}^{\infty} \mathcal{P}_{n}^{+}$. 
Theorem 4.2. Let $\alpha=\left\{\alpha_{k}\right\}_{k=0}^{\infty}$ be a sequence of real numbers, and let $n \in \mathbb{N}$. The following are equivalent.

(i) $U_{\alpha}\left(\mathcal{P}_{n}^{+}\right) \subseteq \mathcal{P}_{n}^{+} \cup\{0\}$;

(ii) $U_{\alpha}\left((1+z)^{n}\right) \in \mathcal{P}_{n}^{+} \cup\{0\}$;

(iii)

$$
\sum_{k=0}^{\lfloor n / 2\rfloor}\left(\sum_{j=0}^{k} \frac{\alpha_{j}}{(k+j) !(k-j) !}\right) \frac{z^{k}}{(n-2 k) !} \in \mathcal{P}_{n}^{+} \cup\{0\} .
$$

Proof. Let $\mu=\left\{\alpha_{0}, 0, \alpha_{1}, 0, \alpha_{2}, \ldots\right\}$, and consider the operator $T_{\mu}$ given by (7). Then $U_{\alpha}=T_{\mu}^{E}$. By the Hermite-Biehler theorem, for each $P \in \mathcal{P}_{n}^{+}$,

$$
T_{\mu}(P) \text { is weakly Hurwitz stable if and only if } U_{\alpha}(P) \in \mathcal{P}_{n}^{+} \text {. }
$$

Now,

$$
P_{\mu, n}(z)=n ! \sum_{k=0}^{\lfloor n / 2\rfloor}\left(\sum_{j=0}^{k} \frac{\alpha_{j}}{(k+j) !(k-j) !}\right) \frac{z^{n-2 k}}{(n-2 k) !},
$$

so $P_{\mu, n}(z)$ is weakly Hurwitz stable or identically zero if and only if (iii) holds. The theorem follows from Theorem 3.2 .

Example 4.3. Let us use Theorem 4.2 to give a second proof of Conjecture 1.1 In this situation $\alpha=\{1,-1,0,0, \ldots\}$, and

$U_{\alpha}\left((1+z)^{n}\right)=\sum_{k=0}^{n}\left(\left(\begin{array}{l}n \\ k\end{array}\right)^{2}-\left(\begin{array}{c}n \\ k-1\end{array}\right)\left(\begin{array}{c}n \\ k+1\end{array}\right)\right) z^{k}=\sum_{k=0}^{n} \frac{1}{n+1}\left(\begin{array}{c}n+1 \\ k\end{array}\right)\left(\begin{array}{l}n+1 \\ k+1\end{array}\right) z^{k}$.

These polynomials are known as the Narayana polynomials. There are numerous proofs that the Narayana polynomials have only real zeros. The simplest is probably based on the Maló Theorem, see e.g. [6, Theorem 2.4].

The corresponding theorem for $V_{\alpha}$ reads as follows.

Theorem 4.4. Let $\alpha=\left\{\alpha_{k}\right\}_{k=0}^{\infty}$ be a sequence of real numbers, and let $n \in \mathbb{N}$. The following are equivalent.

(i) $V_{\alpha}\left(\mathcal{P}_{n}^{+}\right) \subseteq \mathcal{P}_{n}^{+} \cup\{0\}$;

(ii) $V_{\alpha}\left((1+z)^{n}\right) \in \mathcal{P}_{n}^{+} \cup\{0\}$;

$$
\sum_{k=0}^{\lfloor(n-1) / 2\rfloor}\left(\sum_{j=0}^{k} \frac{\alpha_{j}}{(k+1+j) !(k-j) !}\right) \frac{z^{k}}{(n-2 k-1) !} \in \mathcal{P}_{n}^{+} \cup\{0\} .
$$

Proof. Consider $\mu=\left\{0, \alpha_{0}, 0, \alpha_{1}, 0, \ldots\right\}$. The proof proceeds just as the proof of Theorem 4.2, since $V_{\alpha}=T_{\mu}^{O}$.

\section{Transcendental Pólya-Schur characterizations of TRANSFORMATIONS}

In this section we provide transcendental characterizations of various transformations. The following spaces of entire functions are relevant for our purposes.

- $\mathcal{H}(\mathbb{C})$ is the set of entire functions that are limits, uniformly on compact subsets of $\mathbb{C}$, of univariate polynomials that have zeros only in the closed left half-plane; 
- $\mathcal{H}(\mathbb{R})$ is the space of entire functions in $\mathcal{H}(\mathbb{C})$ with real coefficients;

- The Laguerre-Pólya class, $\mathcal{L}-\mathcal{P}$, of entire functions consists of all entire functions that are limits, uniformly on compact subsets of $\mathbb{C}$, of real polynomials with only real zeros. A function $\phi$ is in $\mathcal{L}-\mathcal{P}$ if and only if it can be expressed in the form

$$
\phi(z)=C z^{n} e^{-a z^{2}+b z} \prod_{j=0}^{\infty}\left(1+\rho_{j} z\right) e^{-\rho_{j} z},
$$

where $n \in \mathbb{N}, a, b, c \in \mathbb{R}, a \geq 0$, and $\left\{\rho_{j}\right\}_{j=0}^{\infty} \subset \mathbb{R}$ satisfies $\sum_{j=0}^{\infty} \rho_{j}^{2}<\infty$, see [9, Chapter VIII];

- $\mathcal{L}-\mathcal{P}^{+}$consists of those functions in the Laguerre-Pólya class that have non-negative Taylor coefficients. A function $\phi$ is in $\mathcal{L}-\mathcal{P}^{+}$if and only it can be expressed as

$$
\phi(z)=C z^{M} e^{a z} \prod_{j=0}^{\infty}\left(1+\rho_{j} z\right),
$$

where $a, C \geq 0, M \in \mathbb{N}$ and $\sum_{j=0}^{\infty} \rho_{j}<\infty$, see [9, Chapter VIII].

The following very useful lemma is due to Szász [18].

Lemma 5.1 (Szász). Let $H \subset \mathbb{C}$ be an open half-plane with boundary containing the origin, and let $f(z)=b_{M} z^{M}+b_{M+1} z^{M+1}+\cdots+b_{N} z^{N} \in \mathbb{C}[z]$, where $b_{M} b_{N} \neq 0$. If $f(\zeta) \neq 0$ for all $\zeta \in H$, then

$$
|f(z)| \leq\left|b_{M}\right||z|^{M} \exp \left(\frac{\left|b_{M+1}\right|}{\left|b_{M}\right|}|z|+3|z|^{2} \frac{\left|b_{M+1}\right|^{2}}{\left|b_{M}\right|^{2}}+3|z|^{2} \frac{\left|b_{M+2}\right|}{\left|b_{M}\right|}\right),
$$

for all $z \in \mathbb{C}$.

Remark 5.2. The typical use of Lemma 5.1 is as follows. Suppose that $\left\{P_{n}(z)\right\}_{n=0}^{\infty}$ is a sequence of polynomials that are non-vanishing in $H$, where $H$ is as in Lemma 5.1. Write

$$
P_{n}(z)=\sum_{k=M}^{N_{n}} a_{n, k} z^{k}
$$

and let $\left\{a_{k}\right\}_{k=M}^{\infty}$ be a sequence of complex numbers with $a_{M} \neq 0$. If $\lim _{n \rightarrow \infty} a_{n, k}=$ $a_{k}$ for each $k \geq M$, then there is a subsequence of $\left\{P_{n}(z)\right\}_{n=0}^{\infty}$ converging, uniformly on compact subsets of $\mathbb{C}$, to the entire function $\sum_{k=M}^{\infty} a_{k} z^{k}$. This follows from Montel's theorem, since $\left\{P_{n}(z)\right\}_{n=0}^{\infty}$ is locally uniformly bounded sequence by Lemma 5.1 .

For a proof of the next lemma we refer to [9, Chapter VIII] or [1, Theorem 12].

Lemma 5.3. Let $\phi(z)=\sum_{k=0}^{\infty} a_{k} z^{k} / k$ ! be a formal power series with complex coefficients, and let $H \subset \mathbb{C}$ be an open half-plane with boundary containing the origin. Then $\phi(z)$ is an entire function which is the limit, uniformly on compact subsets of $\mathbb{C}$, of polynomials that are non-vanishing in $H$ if and only if

$$
\phi_{n}(z)=\sum_{k=0}^{n}\left(\begin{array}{l}
n \\
k
\end{array}\right) a_{k} z^{k}
$$

is either identically zero or non-vanishing in $H$, for each $n \in \mathbb{N}$. 
Theorem 5.4. Let $\mu=\left\{\mu_{k}\right\}_{k=0}^{\infty}$ be a sequence of complex numbers and let $\left\{\gamma_{k}\right\}_{k=0}^{\infty}$ be defined by (11). Define a formal power series by

$$
T_{\mu}\left(e^{z}\right)=\sum_{k=0}^{\infty} \frac{\gamma_{k}}{k !} z^{k}
$$

The following are equivalent.

(i) For all polynomials $P(z)$ with only real and non-positive zeros, the polynomial $T_{\mu}(P(z))$ is either identically zero or weakly Hurwitz stable;

(ii) $T_{\mu}\left(e^{z}\right) \in \mathcal{H}(\mathbb{C}) \cup\{0\}$;

(iii) $T_{\mu}\left(\mathcal{L}-\mathcal{P}^{+}\right) \subseteq \mathcal{H}(\mathbb{C}) \cup\{0\}$.

Proof. Note that $\phi_{n}(z)$ is weakly Hurwitz stable if and only if $z^{n} \phi_{n}(1 / z)$ is weakly Hurwitz stable. Combining Theorem 3.1 and Lemma 5.3 yields the equivalence of (i) and (ii). Clearly (iii) $\Rightarrow$ (ii). Assume (i) and let $\phi \in \mathcal{L}-\mathcal{P}^{+}$. Then, by Lemma 5.3. $\phi_{n}(z)$ is a polynomial with only real and non-positive zeros (unless identically zero) for each $n \in \mathbb{N}$. Thus $T_{\mu}\left(\phi_{n}(z / n)\right)$ is weakly Hurwitz stable or identically zero for each $n \geq 1$. Note that

$$
\lim _{n \rightarrow \infty}\left(\begin{array}{l}
n \\
k
\end{array}\right) \frac{a_{k}}{n^{k}}=\frac{a_{k}}{k !},
$$

for each $k \in \mathbb{N}$. By Remark 5.2 , there is a subsequence $\left\{n_{j}\right\}_{j=0}^{\infty}$ such that

$$
\lim _{j \rightarrow \infty} T_{\mu}\left(\phi_{n_{j}}\left(z / n_{j}\right)\right)=T_{\mu}(\phi(z)),
$$

where the convergence is uniform on each compact subset of $\mathbb{C}$. Hence $T_{\mu}(\phi) \in$ $\mathcal{H}(\mathbb{C}) \cup\{0\}$.

Remark 5.5. The characterization of the "good" sequences $\left\{\mu_{k}\right\}_{k=0}^{\infty}$ in Theorem 5.4 is in terms of the sequences $\left\{\gamma_{k}\right\}_{k=0}^{\infty}$. How do we translate between the two sequences? The answer is classical and is called the Chebyshev relation, see [15, p. $54]$ :

if and only if

$$
\gamma_{k}=\sum_{j=0}^{\lfloor k / 2\rfloor}\left(\begin{array}{c}
k \\
j
\end{array}\right) \mu_{k-2 j}, \quad \text { for all } k \in \mathbb{N}
$$

$$
\mu_{k}=\sum_{j=0}^{\lfloor k / 2\rfloor}(-1)^{j} \frac{k}{k-j}\left(\begin{array}{c}
k-j \\
j
\end{array}\right) \gamma_{k-2 j}, \quad \text { for all } k \in \mathbb{N} .
$$

The following lemma follows easily from Remark 5.2 and the Hermite-Biehler Theorem.

Lemma 5.6. Let $\phi(z)$ be formal power series with real and non-negative coeffcients. Then $\phi(z) \in \mathcal{L}-\mathcal{P}^{+}$if and only if $\phi\left(z^{2}\right) \in \mathcal{H}(\mathbb{R})$.

Theorem 5.7. Let $\alpha=\left\{\alpha_{k}\right\}_{k=0}^{\infty}$ be a sequence of real numbers, and let $n \in \mathbb{N}$. The following are equivalent.

(i) $U_{\alpha}\left(\mathcal{P}^{+}\right) \subseteq \mathcal{P}^{+} \cup\{0\}$;

(ii) $U_{\alpha}\left(e^{z}\right) \in \mathcal{L}-\mathcal{P}^{+} \cup\{0\}$, that is,

$$
\sum_{k=0}^{\infty}\left(\sum_{j=0}^{k} \frac{\alpha_{j}}{(k+j) !(k-j) !}\right) z^{k} \in \mathcal{L}-\mathcal{P}^{+} \cup\{0\} ;
$$


(iii) $U_{\alpha}\left(\mathcal{L}-\mathcal{P}^{+}\right) \subseteq \mathcal{L}-\mathcal{P}^{+} \cup\{0\}$.

Proof. Let $\mu=\left\{\alpha_{0}, 0, \alpha_{1}, 0, \alpha_{2}, \ldots\right\}$. Then $U_{\alpha}\left(\mathcal{P}^{+}\right) \subseteq \mathcal{P}^{+} \cup\{0\}$ if and only if $T_{\mu}\left(\mathcal{P}^{+}\right) \subseteq \mathcal{H}(\mathbb{R}) \cup\{0\}$, by the Hermite-Biehler theorem. By Lemma 5.6. $U_{\alpha}\left(e^{z}\right) \in$ $\mathcal{L}-\mathcal{P}^{+}$if and only if $T_{\mu}\left(e^{z}\right) \in \mathcal{H}(\mathbb{R})$, and $U_{\alpha}\left(\mathcal{L}-\mathcal{P}^{+}\right) \subseteq \mathcal{L}-\mathcal{P}^{+} \cup\{0\}$ if and only if $T_{\mu}\left(\mathcal{L}-\mathcal{P}^{+}\right) \subseteq \mathcal{H}(\mathbb{R}) \cup\{0\}$.

The proof of the next theorem is almost identical to that of Theorem 5.7 .

Theorem 5.8. Let $\alpha=\left\{\alpha_{k}\right\}_{k=0}^{\infty}$ be a sequence of real numbers, and let $n \in \mathbb{N}$. The following are equivalent.

(i) $V_{\alpha}\left(\mathcal{P}^{+}\right) \subseteq \mathcal{P}^{+} \cup\{0\}$;

(ii) $V_{\alpha}\left(e^{z}\right) \in \mathcal{L}-\mathcal{P}^{+} \cup\{0\}$, that is,

$$
\sum_{k=0}^{\infty}\left(\sum_{j=0}^{k} \frac{\alpha_{j}}{(k+1+j) !(k-j) !}\right) z^{k} \in \mathcal{L}-\mathcal{P}^{+} \cup\{0\} ;
$$

(iii) $V_{\alpha}\left(\mathcal{L}-\mathcal{P}^{+}\right) \subseteq \mathcal{L}-\mathcal{P}^{+} \cup\{0\}$.

\section{Applications and examples}

Let us apply Theorem 5.7 to a question posed by Fisk [7. For $r \in \mathbb{N}$, let $S_{r}=U_{\alpha}$ where $\alpha_{0}=1, \alpha_{r}=-1$, and $\alpha_{i}=0$ for all $i \notin\{0, r\}$. In other words

$$
S_{r}\left(\sum_{i=0}^{n} a_{i} z^{i}\right)=\sum_{i=0}^{n}\left(a_{i}^{2}-a_{i-r} a_{i+r}\right) z^{i} .
$$

Fisk asked whether $S_{r}\left(\mathcal{P}^{+}\right) \subseteq \mathcal{P}^{+}$for all $r \in \mathbb{N}$. We use Theorem 5.7 and the theory of multiplier sequences to obtain partial results on Fisk's question.

A sequence of real numbers $\left\{\lambda_{k}\right\}_{k=0}^{\infty}$ is a multiplier sequence if for each polynomial $\sum_{k=0}^{n} a_{k} z^{k}$ with only real zeros, the polynomial $\sum_{k=0}^{n} \lambda_{k} a_{k} z^{k}$ is either identically zero or has only real zeros.

Multiplier sequences were characterized in a seminal paper by Pólya and Schur 12. That multiplier sequences preserve the Laguerre-Pólya class follows easily from Remark 5.2 and Lemma [5.3] see also [9, Chapter VIII].

Theorem 6.1 (Pólya and Schur). Let $\left\{\lambda_{k}\right\}_{k=0}^{\infty}$ be a sequence of real numbers, and let $T: \mathbb{R}[z] \rightarrow \mathbb{R}[z]$ be the corresponding (diagonal) linear operator defined by $T\left(z^{k}\right)=\lambda_{k} z^{k}$, for all $k \in \mathbb{N}$. Define $\Phi(z)=T\left(e^{z}\right)$ to be the formal power series

$$
\Phi(z)=\sum_{k=0}^{\infty} \frac{\lambda_{k}}{k !} z^{k} .
$$

The following are equivalent:

(i) $\left\{\lambda_{k}\right\}_{k=0}^{\infty}$ is a multiplier sequence;

(ii) $T(\mathcal{L}-\mathcal{P}) \subseteq \mathcal{L}-\mathcal{P} \cup\{0\}$;

(iii) $\Phi(z)$ defines an entire function which is the limit, uniformly on compact sets, of polynomials with only real zeros of the same sign;

(iv) Either $\Phi(z)$ or $\Phi(-z)$ is an entire function that can be written as

$$
C z^{n} e^{a z} \prod_{k=1}^{\infty}\left(1+\alpha_{k} z\right)
$$


where $n \in \mathbb{N}, C \in \mathbb{R}, a, \alpha_{k} \geq 0$ for all $k \in \mathbb{N}$ and $\sum_{k=1}^{\infty} \alpha_{k}<\infty$;

(v) For all nonnegative integers $n$ the polynomial $T\left[(1+z)^{n}\right]$ has only real zeros of the same sign.

Proposition 6.2. Let $r=0,1,2$ or 3 . Then $S_{r}\left(\mathcal{P}^{+}\right) \subseteq \mathcal{P}^{+} \cup\{0\}$.

Proof. Fix $r \in \mathbb{N}$, and let $S_{r}\left(e^{z}\right)=\sum_{k=0}^{\infty} a_{k, r} z^{k}$. Then

$$
a_{k, r}=\frac{1}{k !(k+r) !}((k+1) \cdots(k+r)-k(k-1) \cdots(k-r+1)) \text {. }
$$

In particular,

$$
S_{1}\left(e^{z}\right)=\sum_{k=0}^{\infty} \frac{1}{k !(k+1) !} z^{k} .
$$

For each $\mu>0$, the sequence $\{1 / \Gamma(k+\mu)\}_{k=0}^{\infty}$ is a multiplier sequence, see [6]. Thus $F_{1}(z) \in \mathcal{L}-\mathcal{P}^{+}$, by Theorem 6.1 This verifies the case when $r=1$ by Theorem 5.7

Next, $a_{k, 2}=(2+4 k) /(k !(k+2) !)$. Both sequences $\{1 /(k+2) !\}_{k=0}^{\infty}$ and $\{2+4 k\}_{k=0}^{\infty}$ are multiplier sequences. Hence, so is $\{(2+4 k) /(k+2) !\}_{k=0}^{\infty}$. The case $r=2$ now follows from Theorems 6.1 and 5.7 .

Since $a_{k, 3}=\left(6+9 k+9 k^{2}\right) /(k !(k+3) !)$, the case when $r=3$ follows from the fact that $\left\{6+9 k+9 k^{2}\right\}_{k=0}^{\infty}$ is a multiplier sequence. Indeed,

$$
\sum_{k=0}^{\infty} \frac{6+9 k+9 k^{2}}{k !} z^{k}=\left(6+18 z+9 z^{2}\right) e^{z}
$$

and the zeros of $6+18 z+9 z^{2}$ are negative. By Theorem $6.1,\left\{6+9 k+9 k^{2}\right\}_{k=0}^{\infty}$ is a multiplier sequence.

Similarly, let $S_{r}^{\prime}=V_{\alpha}$, where $\alpha_{0}=1, \alpha_{r}=-1$, and $\alpha_{i}=0$ for all $i \notin\{0, r\}$.

Proposition 6.3. Let $r=0,1,2$ or 3 . Then $S_{r}^{\prime}\left(\mathcal{P}^{+}\right) \subseteq \mathcal{P}^{+} \cup\{0\}$.

Proof. Fix $r \in \mathbb{N}$, and let $S_{r}^{\prime}\left(e^{z}\right)=\sum_{k=0}^{\infty} b_{k, r} z^{k}$. Then

$$
b_{k, r}=\frac{1}{k !(k+1+r) !}((k+2) \cdots(k+1+r)-k(k-1) \cdots(k-r+1)) .
$$

The proof proceeds as the proof of Proposition 6.2. For example,

$$
b_{k, 3}=\frac{12}{k !(k+4) !}\left(k^{2}+2 k+2\right) \text {. }
$$

The sequence $\left\{k^{2}+2 k+2\right\}_{k=0}^{\infty}$ is a multiplier sequence since

$$
\sum_{k=0}^{\infty} \frac{k^{2}+2 k+2}{k !} z^{k}=\left(2+3 z+z^{2}\right) e^{z}
$$

We conjecture that $S_{r}^{\prime}\left(e^{z}\right) \in \mathcal{L}-\mathcal{P}^{+}$for all $r \in \mathbb{N}$. 
P. BRÄNDÉN

\section{REFined RESUlts on the LOCATION OF ZEROS}

We provide here some general results on the effect on the zeros of polynomials under the transformations $T_{\mu}, U_{\alpha}$ and $V_{\alpha}$.

Theorem 7.1. Let $\mu$ be a sequence of complex numbers, and let

$$
P(z)=1+a_{1} z+\cdots+a_{n} z^{n}=\prod_{j=1}^{n}\left(1+\rho_{j} z\right)
$$

be a complex polynomial of degree $n$. Suppose that $K$ is a circular region containing no zeros of $P_{\mu, n}(z)$. We further require $K$ to be convex if $\mu_{0}=\gamma_{0}=0$. If $\zeta$ is a non-zero complex number for which

$$
\left\{\rho_{i} \zeta+\frac{1}{\rho_{i} \zeta}: 1 \leq i \leq n\right\} \subset K
$$

then $T_{\mu}(P)(\zeta) \neq 0$.

Proof. Let $\zeta$ be as in the statement of the theorem, and suppose that $T_{\mu}(P)(\zeta)=0$. Since

$$
T_{\mu}(P)(\zeta)=W_{\mu, n}\left(\rho_{1} \zeta, \ldots, \rho_{n} \zeta\right)=a_{n} \zeta^{n} \sum_{k=0}^{n} \gamma_{k} e_{n-k}\left(\rho_{1} \zeta+\frac{1}{\rho_{1} \zeta}, \ldots, \rho_{n} \zeta+\frac{1}{\rho_{n} \zeta}\right),
$$

there is, by Theorem 3.1, a $\xi \in K$ such that

$$
0=\sum_{k=0}^{n} \gamma_{k} e_{n-k}(\xi, \ldots, \xi)=\sum_{k=0}^{n} \gamma_{k}\left(\begin{array}{l}
n \\
k
\end{array}\right) \xi^{n-k}=P_{\mu, n}(\xi),
$$

which contradicts the assumptions on $K$.

For $0<\theta<2 \pi$, let $S_{\theta}=\left\{r e^{i \phi}:|\pi-\phi|<\theta\right.$ and $\left.r>0\right\}$ be the sector centered on the negative real axis, and that opens an angle $2 \theta$.

Theorem 7.2. Let $\alpha=\left\{\alpha_{k}\right\}_{k=0}^{\infty}$ be a sequence of real number such that

$$
U_{\alpha}\left((1+z)^{n}\right) \in \mathcal{P}_{n}^{+} .
$$

Suppose that $P(z)=\sum_{k=0}^{n} a_{k} z^{k}$ has zeros only in $S_{\theta}$, where $0 \leq \theta<\pi / 2$. Then $U_{\alpha}(P(z)) \equiv 0$, or all zeros of $U_{\alpha}(P(z))$ are in $S_{2 \theta}$.

Proof. Suppose that $P(z)=\sum_{k=0}^{n} a_{k} z^{k}$ has zeros only in $S_{\theta}$. Write $P(z)=$ $C \prod_{j=1}^{n}\left(1+\rho_{j} z\right)$. Then $\left|\arg \left(\rho_{j}\right)\right|<\theta$ for all $1 \leq j \leq n$. If $|\arg (\zeta)|<\pi / 2-\theta$, then

$$
\left\{\rho_{j} \zeta+\frac{1}{\rho_{j} \zeta}: 1 \leq j \leq n\right\} \subset\{z \in \mathbb{C}: \operatorname{Re}(z)>0\} .
$$

By Theorem 7.1 $T_{\mu}(P)(\zeta) \neq 0$, where $\mu=\left\{\alpha_{0}, 0, \alpha_{1}, 0, \ldots\right\}$. Thus $U_{\alpha}(z)=$ $T_{\mu}(\sqrt{z}) \neq 0$, whenever $|\arg (z)|<\pi-2 \theta$.

The proof of the corresponding theorem for $V_{\alpha}$ is almost identical.

Theorem 7.3. Let $\alpha=\left\{\alpha_{k}\right\}_{k=0}^{\infty}$ be a sequence of real numbers such that

$$
V_{\alpha}\left((1+z)^{n}\right) \in \mathcal{P}_{n}^{+} \text {. }
$$

Suppose that $P(z)=\sum_{k=0}^{n} a_{k} z^{k}$ has zeros only in $S_{\theta}$, where $0 \leq \theta<\pi / 2$. Then $V_{\alpha}(P(z)) \equiv 0$, or all zeros of $V_{\alpha}(P(z))$ are in $S_{2 \theta}$. 


\section{Iterated Turán inequalities and the Boros-Moll Conjecture}

For Taylor coefficients of functions in $\mathcal{L}-\mathcal{P}^{+}$, inequalities stronger than logconcavity hold. Namely the Turán inequalities: If $\sum_{k=0}^{\infty} \gamma_{k} z^{k} / k ! \in \mathcal{L}-\mathcal{P}^{+}$, then the sequence $\left\{\gamma_{k}\right\}_{k=0}^{\infty}$ is log-concave. Craven and Csordas [5] studied iterated Turán inequalities. Define a transformation, $\mathcal{T}$, on infinite sequences as follows. If $\left\{\gamma_{k}\right\}_{k=0}^{\infty}$ is a sequence, let $\mathcal{T}\left(\left\{\gamma_{k}\right\}\right)=\left\{\mu_{k}\right\}_{k=0}^{\infty}$, where $\mu_{k}=\gamma_{k+1}^{2}-\gamma_{k} \gamma_{k+2}$. Note the shift of indices. Craven and Csordas stated the following problem.

Problem 8.1. Let $\sum_{k=0}^{\infty} \gamma_{k} z^{k} / k ! \in \mathcal{L}-\mathcal{P}^{+}$. Is $\mathcal{T}^{i}\left(\left\{\gamma_{k}\right\}\right)$ a non-negative sequence for all $i \in \mathbb{N}$ ?

Craven and Csordas [5] proved that $\mathcal{T}^{2}\left(\left\{\gamma_{k}\right\}\right)$ is non-negative if $\sum_{k=0}^{\infty} \gamma_{k} z^{k} / k ! \in$ $\mathcal{L}-\mathcal{P}^{+}$, and that $\mathcal{T}^{3}\left(\left\{\gamma_{k}\right\}\right)$ is non-negative if $\sum_{k=0}^{\infty} \gamma_{k} z^{k} / k ! \in \mathcal{L}-\mathcal{P}^{+}$and $\gamma_{0}=\gamma_{1}=$ 0 . The second result can be stated as follows: If $\sum_{k=0}^{\infty} \gamma_{k} z^{k} /(k+2) ! \in \mathcal{L}-\mathcal{P}^{+}$, then $\left\{\gamma_{k}\right\}_{k=0}^{\infty}$ is 3 -fold log-concave. In [4] they posed the following problem.

Problem 8.2. Characterize the sequences $\left\{\gamma_{k}\right\}_{k=0}^{\infty}$ such that

$$
\sum_{k=0}^{\infty} \frac{\gamma_{k}}{k !} z^{k} \in \mathcal{L}-\mathcal{P}^{+} \quad \text { and } \quad \sum_{k=0}^{\infty} \frac{t_{k}}{k !} z^{k} \in \mathcal{L}-\mathcal{P}^{+},
$$

where $\left\{t_{k}\right\}_{k=0}^{\infty}=\mathcal{T}\left(\left\{\gamma_{k}\right\}_{k=0}^{\infty}\right)$.

Theorem 5.7 provides a large class of entire functions for which Problem 8.2 holds.

Proposition 8.3. If $\sum_{k=0}^{\infty} \gamma_{k} z^{k} \in \mathcal{L}-\mathcal{P}^{+}$, then $\left\{\gamma_{k}\right\}_{k=0}^{\infty}$ satisfies both conditions in Problem 8.2.

Proof. Since $\{1 / k !\}_{k=0}^{\infty}$ is a multiplier sequence, Theorem6.1 implies $\sum_{k=0}^{\infty} \gamma_{k} z^{k} / k ! \in$ $\mathcal{L}-\mathcal{P}^{+}$. We claim that $\{1 /(k-1) !\}_{k=0}^{\infty}$, where $1 /(-1) !:=0$, is a multiplier sequence. Indeed

$$
\sum_{k=0}^{\infty} \frac{1}{(k-1) ! k !} z^{k}=z \sum_{k=0}^{\infty} \frac{1}{k !(k+1) !} z^{k} \in \mathcal{L}-\mathcal{P}^{+}
$$

By Theorem 5.7 .

$$
\sum_{k=0}^{\infty}\left(\gamma_{k}^{2}-\gamma_{k-1} \gamma_{k+1}\right) z^{k} \in \mathcal{L}-\mathcal{P}^{+}
$$

and by Theorem 6.1

$$
\sum_{k=0}^{\infty} \frac{\gamma_{k}^{2}-\gamma_{k-1} \gamma_{k+1}}{(k-1) !} z^{k}=z \sum_{k=0}^{\infty} \frac{t_{k}}{k !} z^{k} \in \mathcal{L}-\mathcal{P}^{+} .
$$

Let us describe the initial conjecture that motivated Boros and Moll to study infinitely $\log$-concave sequences. For $\ell, m \in \mathbb{N}$ with $\ell \leq m$, let

$$
d_{\ell}(m)=2^{-2 m} \sum_{k=\ell}^{m} 2^{k}\left(\begin{array}{c}
2 m-2 k \\
m-k
\end{array}\right)\left(\begin{array}{c}
m+k \\
m
\end{array}\right)\left(\begin{array}{l}
k \\
\ell
\end{array}\right) .
$$


It is not trivial (at least without the use of computers) to prove that $d_{\ell}(m)$ is the $\ell$ th Taylor coefficient of the polynomial, defined for $a>-1$, by

$$
P_{m}(a)=\frac{2^{m+3 / 2}(a+1)^{m+1 / 2}}{\pi} \int_{0}^{\infty} \frac{1}{\left(x^{4}+2 a x^{2}+1\right)^{m+1}} d x .
$$

Based on computer experiments, Boros and Moll made the following conjecture, see [2].

Conjecture 8.4. For each $m \in \mathbb{N}$, the sequence $\left\{d_{\ell}(m)\right\}_{\ell=0}^{m}$ is infinitely logconcave.

Kauers and Paule $\left[8\right.$ were able to prove log-concavity of $\left\{d_{\ell}(m)\right\}_{\ell=0}^{m}$, using computer algebra. We make the following conjecture.

Conjecture 8.5. For each $m \in \mathbb{N}$, the polynomial

$$
Q_{m}(z)=\sum_{\ell=0}^{m} \frac{d_{\ell}(m)}{\ell !} z^{\ell}
$$

has only real zeros.

We also make a stronger conjecture.

Conjecture 8.6. For each $m \in \mathbb{N}$, the polynomial

$$
R_{m}(z)=\sum_{\ell=0}^{m} \frac{d_{\ell}(m)}{(\ell+2) !} z^{\ell}
$$

has only real zeros.

Note that $Q_{m}(z)=\left(d^{2} / d z^{2}\right)\left(z^{2} R_{m}(z)\right)$, so Conjecture 8.5 is stronger than Conjecture 8.6. The relevance of these conjectures stems from the results of Craven and Csordas on Problem 8.1. If Conjecture 8.5 is true, then $\left\{d_{\ell}(m)\right\}_{\ell=0}^{m}$ is 2-fold log-concave. If Conjecture 8.6 is true, then $\left\{d_{\ell}(m)\right\}_{\ell=0}^{m}$ is 3-fold log-concave.

Acknowledgments. I thank the anonymous referee for carefully reading the paper.

\section{REFERENCES}

[1] J. Borcea, P. Brändén, Pólya-Schur master theorems for circular domains and their boundaries, Ann. of Math. (2) 170, No. 1, (2009), 465-492.

[2] G. Boros, V. Moll, Irresistible integrals, Cambridge Univ. Press, Cambridge, 2004.

[3] D. Cardon, A. Rich, Turán inequalities and subtraction free expressions, J. Inequal. Pure Appl. Math. 9, (2008), no. 4, Article 91, 11pp. (electronic).

[4] T. Craven, G. Csordas, Jensen polynomials and the Turán and Laguerre inequalities, Pacific J. Math. 136 (1989), 241-260.

[5] T. Craven, G. Csordas, Iterated Laguerre and Turán inequalities, J. Inequal. Pure Appl. Math. 3, (2002), no. 3, Article 39, 14pp. (electronic).

[6] T. Craven, G. Csordas, Composition theorems, multiplier sequences and complex zero decreasing sequences, in Value Distribution Theory and Related Topics, Advances in Complex Analysis and Its Applications, Vol. 3, eds. G. Barsegian, I. Laine and C. C. Yang, Kluwer Press, 2004.

[7] S. Fisk, Questions about determinants and polynomials, arXiv:0808.1850

[8] M. Kauers, P. Paule, A computer proof of Moll's log-concavity conjecture, Proc. Amer. Math. Soc. 135, (2007), 3847-3856.

[9] B. Ja. Levin, Distribution of zeros entire functions, Transl. Math. Mono. Vol. 5, Amer. Math. Soc., Providence, RI, 1964; revised ed. 1980. 
[10] P. R. W. McNamara, B. E. Sagan, Infinite log-concavity: developments and conjectures, Adv. Appl. Math. 44 (2010), 1-15.

[11] G. Nikolov, R. Uluchev, Inequalities for real-root polynomials. Proof of a conjecture of Foster and Krasikov, Approximation theory: a volume dedicated to Borislav Bojanov, 201-216, Prof. M. Drinov Acad. Publ. House, Sofia, 2004.

[12] G. Pólya, I. Schur, Über zwei Arten von Faktorenfolgen in der Theorie der algebraischen Gleichungen, J. Reine Angew. Math. 144 (1914), 89-113.

[13] Q. I. Rahman, G. Schmeisser, Analytic Theory of Polynomials, Clarendon Press, Oxford, 2002.

[14] E. D. Rainville, Special functions, The MacMillan Co., New York, 1960.

[15] J. Riordan, Combinatorial identities, John Wiley \& Sons, Inc., New York, 1968.

[16] R. P. Stanley, Enumerative combinatorics. Vol. 2., Cambridge University Press, Cambridge, 1999.

[17] R. P. Stanley, Personal communication, 2008.

[18] O. Szász, On sequences of polynomials and the distribution of their zeros, Bull. Amer. Math. Soc. 49 (1943), 377-383.

Department of Mathematics, Stockholm University, SE-106 91 Stockholm, Sweden

E-mail address: pbranden@math.su.se 